\title{
Medical students' attitudes towards terminology of obesity
}

\author{
Authors: Nora Rako ${ }^{1}$, Hanna Pašić ${ }^{1}$, Maja Baretić ${ }^{1,2}$, Martina Matovinović ${ }^{2}$ \\ ${ }^{1}$ School of Medicine, Medical studies in English, University of Zagreb, Croatia \\ ${ }^{2}$ Department of Endocrinology and Diabetes, Internal Clinic, University Hospital Centre Zagreb, \\ Croatian Obesity Treatment Referral Centre
}

DOI: https://doi.org/10.26800/LV-142-supp5-46

\section{Introduction:}

Obesity, one of the most common health problems encountered in medicine with implications in various other health issues, is nowadays omnipresent. Terminology describing diagnosis of obesity might be considered rude or offensive. Since perception of obese people is sometimes associated with negative attitudes and stereotypes, proper terminology used by physicians is crucial.

Aim:

Study was conducted with the aim to provide a term that would regain consciousness about medical conditions avoiding unnecessary discomfort.

\section{Materials \& Methods:}

Total of 200 students $(77 \%$ females, $23 \%$ males, median age was 18 in a range of $18-30$, median BMI of participants was $23.6 \mathrm{~kg} / \mathrm{m}^{2}$ ) attending final years at the School of Medicine, University of Zagreb answered online questionnaire developed by Croatian Obesity Treatment Referral Centre using SurveyMonkey® tool. Four terms describing excess body weight were evaluated. Attitudes of young people, who are involved in the healthcare system but are without burden of former experience, were analyzed as whether acceptable or unacceptable in healthcare and everyday settings.

\section{Results:}

Participants found terms 'adipose' (adipozan) and 'obese' (pretio) acceptable and don't differentiate them while communicating in healthcare and everyday settings. Term 'chubby' (bucko) was mostly found unacceptable, especially in healthcare settings. Term 'fat' (debeo) was also considered inappropriate in everyday settings, while in the healthcare settings it was found unacceptable by half of the students. Female students considered it inappropriate in interaction with healthcare workers. It was considered inappropriate by most of the overweight students.

\section{Conclusion:}

According to the findings of the survey, the recommendation is to use the terms 'adipose' (adipozan) and 'obese' (pretio), and to avoid colloquial terms, like 'chubby' (bucko). The term 'fat' (debeo) should be used with caution because it could be perceived offensive in healthcare settings. Developing communication skills is fundamental in clinical practice to succeed, especially while interacting with obese people.

Keywords: communication; everyday setting; healthcare setting; obesity; terminology 